Revista Brasileira de Agricultura Irrigada v.7, nº.2, p. 136 - 145, 2013

ISSN 1982-7679 (On-line)

Fortaleza, CE, INOVAGRI - http://www.inovagri.org.br

DOI: $10.7127 /$ rbai.v7n200013

Protocolo 013.13 - 07/03/2013 Aprovado em 25/04/2013

\title{
DEMANDA HÍDRICA DE FRUTEIRAS UTILIZANDO COEFICIENTE DE REDUÇÃO ADEQUADO AO PERÍMETRO IRRIGADO BAIXO ACARAÚ - CE
}

Angela Jessyka Pereira Brito Fontenele ${ }^{1}$; Marco Antônio Rosa de Carvalho², Lilian Cristina Castro de Carvalho ${ }^{2}$; Raimundo Nonato Farias Monteiro ${ }^{3}$; Alexsandro Oliveira da Silva ${ }^{3}$; Aluízio Tavares Cordeiro Neto ${ }^{4}$.

\section{RESUMO}

Este trabalho foi realizado com o objetivo de avaliar a adequação dos modelos de estimativa do coeficiente de cobertura $(\mathrm{Kr})$, bem como estabelecer uma equação adequada para a região do Baixo Acaraú no estado do Ceará. As coletas foram realizadas em três lotes dentro do Perímetro de Irrigação no mês de março de 2010 em cultivos irrigados por microaspersão nas seguintes culturas: coco, graviola, laranja, manga e sapoti. Foram feitas 60 medições de diâmetro da copa, que aplicadas aos índices de cobertura (Cs), determinaram os coeficientes de cobertura $(\mathrm{Kr})$ por meio de diferentes modelos propostos na literatura. $\mathrm{O}$ delineamento experimental foi o inteiramente aleatorizado, sendo considerado tratamento cada equação de $\mathrm{Kr}$ (sete) e repetição cada cultura (cinco). O trabalho mostrou que não existe diferença significativa entre as equações de determinação do $\mathrm{Kr}$, quando aplicadas às culturas na fase adulta. A equação ajustada para a estimativa do coeficiente de redução da evapotranspiração $(\mathrm{Kr}),(\mathrm{Kr}=1,1226 . \mathrm{Cs}+0,0434)$, apresentou resultados semelhantes aos encontrados quando utilizada a metodologia proposta por Keller \& Karmeli. O período de maior demanda hídrica se encontra no segundo semestre do ano, devendo-se dimensionar os sistemas de irrigação com base neste período.

Palavras-chave: irrigação localizada, índice de cobertura do solo, evapotranspiração

\section{WATER DEMAND OF FRUIT CROPS USING SUITABLE REDUCTION COEFFICIENT FOR BAIXO ACARAÚ IRRIGATED AREA, LOCATED IN CEARÁ.}

\author{
ABSTRACT

\footnotetext{
${ }^{1}$ Mestranda em Engenharia Agrícola, UFRPE. Rua Dom Manuel Medeiros, s/n, Dois Irmãos, 52171-900, Recife, PE. jessykafontenele@ hotmail.com.

${ }^{2}$ Professor do Instituto Federal do Ceará, IFCE. Av. Dr. Guarani, 317, Derby Clube, 62042-030, Sobral, CE. marcorosa@ifce.edu.br, liliancarv@ifce.edu.br.

${ }^{3}$ Doutorando em Agronomia (Irrigação e Drenagem), FCA/UNESP. Rua José Barbosa de Barros, Jd Paraíso, 18610-510, Botucatu, SP, raimundomonteiro@fca.unesp.br, alexsandro01@ @ fca.unesp.br .

${ }^{4}$ Mestre em Engenharia Agrícola, UFRPE, Rua Dom Manuel Medeiros, s/n, Dois Irmãos, 52171-900, Recife, PE. aluizioten@ hotmail.com.
} 


\section{DEMANDA HÍDRICA DE FRUTEIRAS UTILIZANDO COEFICIENTE DE REDUÇÃO ADEQUADO AO PERÍMETRO IRRIGADO BAIXO ACARAÚ - CE}

This work was done with the objective of evaluating the adaptation of the models in estimating a reduction factor $(\mathrm{Kr})$ as well as to establish an appropriate equation for the region of the estimation models as well as to establish a suitable equation for the Baixo Acaraú in the state of Ceará. The data collections were accomplished in three lots inside of the Irrigation Perimeter in March 2010 in micro sprinkler irrigated crops on the following crops: coconut, soursop, orange, mango and sapodilla. 60 measurements were made of canopy diameter, which applied to coverage ratios $(\mathrm{Cs})$, determined the coverage ratio $(\mathrm{Kr})$ through different models proposed in the literature. The experimental design was completely randomized, and it was considered treatment each equation of $\mathrm{Kr}$ (five) and repetition every culture (seven). The study showed that there is not significant difference between the equations of determination of $\mathrm{Kr}$ when applied to crops in adulthood. The fitted equation to estimate evapotranspiration reduction coefficient $(\mathrm{Kr}),(\mathrm{Kr}=1.1226$. $\mathrm{Cs}+0.0434)$ showed similar results to those found when using the methodology proposed by Keller \& Karmeli. The period of greatest water demand is in the second half of the year, and it should scale irrigation systems based on this period.

Keywords: localized irrigation, ground cover, evapotranspiration

\section{INTRODUÇÃO}

A água doce é um recurso finito essencial para a conservação da vida além de ser um dos principais fatores de produção na agricultura, sendo essencial para o desenvolvimento sustentável da atividade agrícola. Segundo AGRIANUAL (2007), no ano compreendido entre o segundo semestre de 2003 e o primeiro de 2004, a agricultura irrigada respondeu por $44 \%$ do total de alimentos produzido no mundo. Nesse período, o uso da água na agricultura representou em nível mundial, $70,2 \%$ de toda a água doce, enquanto a indústria utilizou 20,3\% e o abastecimento humano ficou com apenas 9,5\%. As proporções acima mostram que os irrigantes, principais usuários da água, não podem prescindir da mais alta eficiência possível na utilização da mesma.

Apesar do grande consumo de água, a irrigação representa a maneira mais eficiente de aumento da produção de alimentos e desenvolve um papel primordial no aspecto econômico e social, além de suprir irregularidades pluviométricas (FRIZZONE \& ANDRADE JÚNIOR, 2005). Em uma região como a do Nordeste brasileiro em que a taxa de evapotranspiração pode chegar a 3 vezes o valor da taxa de precipitação, torna-se indispensável a utilização da irrigação para a produção agrícola, contudo, devido a limitada disponibilidade hídrica desta região é necessário sistemas de irrigação que aproveitem o máximo uso da água. Dentre os sistemas de irrigação utilizados a irrigação localizada destaca-se nesse contexto, principalmente devido a sua eficiência na distribuição de água no solo, próximo ao sistema radicular das plantas e no uso da fertirrigação, permitindo economia de mão de obra. Sendo assim, outros métodos se tornam menos indicados devido ao consumo maior de água (DOGAN et al., 2007; HERA et al., 2007) e menor eficiência do sistema.

Dentre os principais perímetros irrigados do estado do Ceará, o perímetro irrigado do Baixo Acaraú destaca-se por ser um dos mais modernos, possuindo uma área irrigada de 8.816,61 ha, sendo a principal fonte hídrica o rio Acaraú, dentre os principais cultivos deste perímetro destacam-se a produção de frutas que ocupam uma área agrícola de 1.947,5 ha 


\section{DEMANDA HÍDRICA DE FRUTEIRAS UTILIZANDO COEFICIENTE DE REDUÇÃO ADEQUADO AO PERÍMETRO IRRIGADO BAIXO ACARAÚ - CE}

(ADECE, 2012), sendo irrigados pelo sistema de irrigação localizada (gotejamento e microaspersão) em função da economia de água propiciada (ALLEN \& PEREIRA, 2009; HERA et al., 2007; KLAR, 1991). Devido a grande área de fruticultura irrigada que este perímetro apresenta, é de grande importância utilizar um adequado manejo da irrigação, visando à economia de água nesta atividade agrícola.

A área molhada junto às plantas é uma das características do sistema de irrigação localizada e é de grande importância para o seu dimensionamento, manejo e sucesso do agronegócio. Keller \& Karmeli (1974) recomendam áreas molhadas de $20 \%$ a $30 \%$ para regiões úmidas e semiáridas. Portanto é de suma importância ter a estimativa específica do fator de redução da evapotranspiração $(\mathrm{Kr})$ para dimensionar com melhor precisão o

\section{MATERIAL E MÉTODOS}

Este trabalho foi desenvolvido em três lotes distintos no Perímetro de Irrigação do Baixo Acaraú no Estado do Ceará, pertencente ao Departamento Nacional de Obras Contra as Secas DNOCS, com sede situada no município de Marco (latitude: 03 ${ }^{\circ}$ 07'S, longitude: $40^{\circ} 08^{\prime} \mathrm{W}$ e altitude: $56 \mathrm{~m}$ ), distante $220 \mathrm{~km}$ de Fortaleza, no mês de março de 2010. A evapotranspiração de referência foi estimada pelo método Penman-MonteithFAO56 (ALLEN et al., 1998), com dados obtidos diariamente em estação automatizada disponíveis no site do Instituto Nacional de Meteorologia (INMET) no período de 1 de janeiro de 2010 a 31 de dezembro de 2010 simulando o consumo de água pelas culturas durante o ano.

As culturas para as quais se realizaram as pesquisas foram: coco (Cocos nucifera) 42 meses de plantio, sistema de irrigação, sabendo exatamente a quantidade de água aplicada a cada turno. Desta maneira, realizar avaliações visando à adequação dos modelos de estimativa do coeficiente de cobertura $(\mathrm{Kr})$, bem como estabelecer uma equação adequada para cada região é de fundamental importância, otimizando assim, a produção e viabilizando tanto no aspecto agronômico quanto no econômico a produção agrícola (FRIZZONE \& ANDRADE JÚNIOR, 2005; ANDRADE JÚNIOR et al., 2001).

O objetivo desde trabalho foi avaliar a adequação de modelos de estimativa do coeficiente de cobertura da evapotranspiração $(\mathrm{Kr})$ para diferentes culturas, bem como estabelecer uma equação adequada para o Perímetro Irrigado do Baixo Acaraú, além de estimar o consumo hídrico de diversas culturas para esta região contribuindo para o uso racional da água na irrigação.

graviola (Annona muricata) 60 meses de plantio, laranja (Citrus sinensis) 30 meses

de plantio, manga (Mangifera indica) 86 meses de plantio e sapoti (Manilkara achras) 61 meses de plantio.

Em todos os pomares foram escolhidas, ao acaso, 4 plantas e determinadas, com 3 repetições, medidas do diâmetro da projeção da copa na superfície do solo, conforme Carvalho et al. (2007), no intervalo entre 11 e 13 horas do dia. Com os valores médios dos diâmetros das copas (D), foram determinados, para todas as culturas, os índices de cobertura (Cs), que expressam a fração da superfície do solo sombreada pela planta, fornecidos pela equação (1).

$$
C S=\frac{A}{E p \times E f}
$$




\section{DEMANDA HÍDRICA DE FRUTEIRAS UTILIZANDO COEFICIENTE DE REDUÇÃO ADEQUADO AO PERÍMETRO IRRIGADO BAIXO ACARAÚ - CE}

em que,

A - área de projeção da copa, $\mathrm{m}^{2}$;

Ep - Espaçamento entre plantas, m;

Ef - Espaçamento entre fileiras, $\mathrm{m}$;

Para a determinação do coeficiente de redução da evapotranspiração $(\mathrm{Kr})$ em cada cultura foram utilizada sete fórmulas empíricas algumas citadas por Mantovani et al. (2009) e outras obtidas em Carvalho et al. (2007) e Ferreira et al. (2010) conforme a Tabela 1:

Tabela 1. Metodologias de cálculo de coeficiente de redução da evapotranspiração (Kr).

\begin{tabular}{clcc}
\hline Metodologia & \multicolumn{1}{c}{ Equações } & Fontes \\
\hline Aljibury & $\mathrm{Kr}_{\mathrm{A}}=1,34 . \mathrm{Cs}$ & $(2)$ & Gomes (1997) \\
Decroix & $\mathrm{Kr}_{\mathrm{D}}=0,1+\mathrm{Cs}$ ou 1, & $(3)$ & Pizarro Cabello (1996) \\
Freeman/Garzoli & $\mathrm{Kr}_{\mathrm{F}}=\mathrm{Cs}+0,5 .(1-\mathrm{Cs})$ & $(4)$ & Vermeiren \& Jobling (1997) \\
Keller & $\mathrm{Kr}_{\mathrm{K}}=\mathrm{Cs}+0,15 .(1-\mathrm{Cs})$ & $(5)$ & Gomes (1997) \\
Keller / Karmeli & $\mathrm{Kr}_{\mathrm{KK}}=\mathrm{Cs} / 0,85$ ou 1 & $(6)$ & Keller \& Karmeli (1974) \\
Carvalho et al. & $\mathrm{Kr}_{\mathrm{C}}=1,1451 . \mathrm{Cs}+0,0273$ & $(7)$ & Carvalho et al. (2007) \\
Ferreira et al. & $\mathrm{Kr}_{\mathrm{F}}=1,0224 . \mathrm{Cs}+0,1149$ & $(8)$ & Ferreira et al. (2010)
\end{tabular}

$\mathrm{Na}$ equação (4) recomenda-se tomar $\mathrm{Kr}=\mathrm{Cs}$ quando $\mathrm{Cs}<0,5$ e $\mathrm{Kr}=1$ quando $\mathrm{Cs}=1$. A evapotranspiração reduzida da cultura (ETr) foi calculada conforme descrito por Mantovani e al. (2009) e Allen et al. (2009) através da equação:

$$
E T r=E T O X K C \times K r
$$

Em que:

ETo - Evapotranspiração de referencia, $\mathrm{mm} \mathrm{dia}{ }^{-1}$;
Kc - Coeficiente de cultivo adimensional;

$\mathrm{Kr}$ - coeficiente de redução da evapotranspiração, adimensional.

Os valores de Kc utilizados encontram-se dispostos na Tabela 2. Devido à falta de estudos locais sobre o manejo da irrigação e o consumo de água na fruticultura foram usados coeficientes de cultivo de regiões com climas semelhantes ou como referência estudos obtidos no boletim FAO 56 (DOORENBOS \& PRUIT, 1997), para haver uma aproximação dos valores reais da região.

Tabela 2. Coeficiente de cultivo $(\mathrm{Kc})$ das culturas com suas respectivas fontes

\begin{tabular}{ccc}
\hline Cultura & Kc & Fonte \\
\hline Coco & 0,95 & Fontes et al. (2002) \\
Graviola & 0,85 & Silva (2003) \\
Laranja & 0,8 & Doorenbos \& Pruit (1997) \\
Manga & 1 & Doorenbos \& Pruit (1997) \\
Sapoti & 0,7 & Gondim et al. (2008) \\
\hline
\end{tabular}




\section{DEMANDA HÍDRICA DE FRUTEIRAS UTILIZANDO COEFICIENTE DE REDUÇÃO ADEQUADO AO PERÍMETRO IRRIGADO BAIXO ACARAÚ - CE}

O volume de água a ser aplicado foi estimado pela seguinte expressão como feito por Ferreira et al. (2010):

$$
V a=E \operatorname{Tr} \times A \times \operatorname{Tr}
$$

\section{RESULTADOS E DISCUSSÃO}

A Tabela 3 apresenta os valores de espaçamento, diâmetro da copa e coeficiente de cobertura para as culturas medidas no perímetro irrigado. Observa-se
Va - volume de água a ser aplicado por planta, L;

A - área ocupada por planta, $\mathrm{m}^{2}$; e, $\mathrm{Tr}$ - turno de irrigação, dia (utilizou-se $\mathrm{Tr}$ $=1$ ).

que o maior valor de $\mathrm{Cs}$ foi encontrado para a graviola enquanto a cultura da laranja apresentou o menor valor de cobertura do solo.

Tabela 3 - Espaçamento em campo, diâmetro da copa e coeficiente de cobertura (Cs)

\begin{tabular}{lccc}
\hline Cultura & Espaçamento $(\mathbf{m})$ & Diâmetro $(\mathbf{m})$ & Cs \\
\hline Coco & $7 \times 7$ & 4,94 & 0,39 \\
Graviola & $6 \times 6$ & 5,89 & 0,76 \\
Laranja & $6 \times 5$ & 3,45 & 0,31 \\
Manga & $6 \times 8$ & 4,99 & 0,41 \\
Sapoti & $6 \times 4$ & 3,97 & 0,52 \\
\hline
\end{tabular}

A análise de variância mostrou que não houve diferença significativa entre os tratamentos mostrados na Tabela 4 para um nível de significância de $5 \%$, com coeficiente de variação de $35,6 \%$, coerente com o indicado por Vieira (2006), que considera bom um ensaio de campo com coeficiente de variação próximo de $30 \%$. Utilizando as equações 2, 3, 4, 5 e 6 para determinação do $\mathrm{Kr}$, Carvalho et al. (2007), não encontraram diferenças significativas para um nível de $5 \%$ e o coeficiente de variação do ensaio foi de $32,6 \%$, mesmo trabalhando com oito culturas diferentes (açaí, acerola, ata, caju, goiaba, graviola pitanga e sapoti).

Resultados semelhantes podem ser observados em Ferreira et al. (2010), em estudos sobre a demanda hídrica e a utilização do coeficiente de redução da evapotranspiração, na região do Baixo Jaguaribe no Ceará.

Tabela 4. Coeficiente de redução da evapotranspiração $(\mathrm{Kr})$, por diferentes metodologias Coeficiente de redução da evapotranspiração $(\mathrm{Kr})$

\begin{tabular}{llllllll}
\hline Cultura & $\mathrm{Kr}_{\mathrm{A}}$ & $\mathrm{Kr}_{\mathrm{D}}$ & $\mathrm{Kr}_{\mathrm{F}}$ & $\mathrm{Kr}_{\mathrm{K}}$ & $\mathrm{Kr}_{\mathrm{KK}}$ & $\mathrm{Kr}_{\mathrm{C}}$ & $\mathrm{Kr}_{\mathrm{F}}$ \\
\hline Coco & 0,52 & 0,49 & 0,39 & 0,48 & 0,46 & 0,47 & 0,51 \\
Graviola & 1,00 & 0,86 & 0,88 & 0,80 & 0,89 & 0,89 & 0,89 \\
Laranja & 0,42 & 0,41 & 0,31 & 0,41 & 0,36 & 0,38 & 0,43 \\
Manga & 0,55 & 0,51 & 0,41 & 0,50 & 0,48 & 0,49 & 0,53 \\
Sapoti & 0,70 & 0,62 & 0,76 & 0,59 & 0,61 & 0,62 & 0,64 \\
\hline
\end{tabular}




\section{DEMANDA HÍDRICA DE FRUTEIRAS UTILIZANDO COEFICIENTE DE REDUÇÃO ADEQUADO AO PERÍMETRO IRRIGADO BAIXO ACARAÚ - CE}

Com os dados apresentados na Tabela 4, foi feito um modelo de regressão linear com as sete equações $(2,3,4,5,6,7$ e 8), que forneceu uma equação ajustada $(\mathrm{Kr}=1,1226 . \mathrm{Cs}+0,0434) \mathrm{com}$ um coeficiente de determinação $\mathrm{R}^{2}=0,92$, como mostra a Figura 1A. Carvalho et al. (2007), estudando coeficientes de cobertura em cultivo de fruteiras microirrigadas, apresentaram valores semelhantes de $\mathrm{Kr}$ para as coberturas do solo das culturas estudadas (Figura 1B). Comparando-se os valores de Cs para a cultura do melão entre diferentes métodos, Dutra et al. (2000), observaram que não houve diferenças entre os valores encontrados aos estimados utilizando Keller \& Karmeli (1974). Ferreira et al. (2010) avaliando a adequação de modelos de estimativa de coeficiente de redução da evapotranspiração $(\mathrm{Kr})$ para diferentes culturas na região do Baixo Jaguaribe no estado do Ceará, afirmam que não houve diferenças significativas entre as equação de $\mathrm{Kr}$ estudadas para as culturas na fase adulta, sendo a equação ajustada no trabalho, semelhantes as propostas por Keller \& Karmeli (1974) e Vermein \& Jobling (1997).
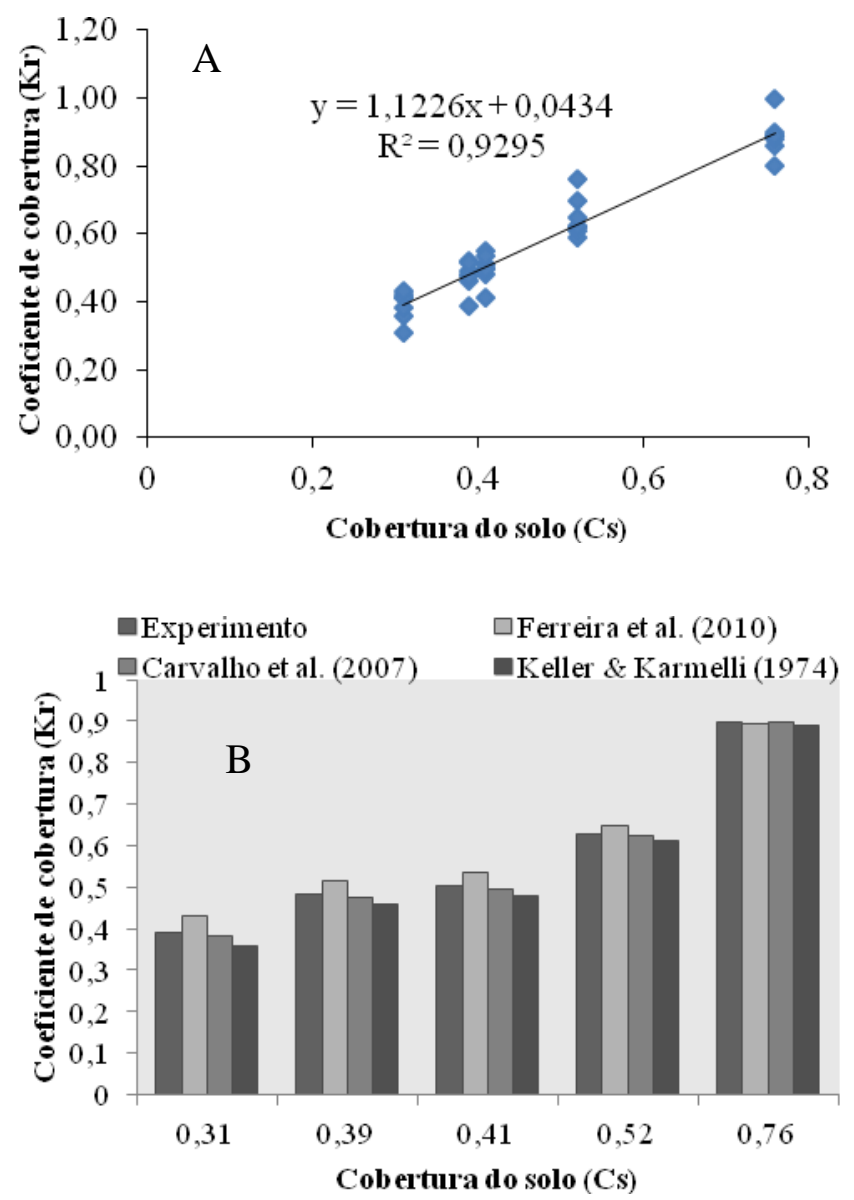

Figura 1. Coeficiente de cobertura $(\mathrm{Kr})$ gerado utilizando a regressão das sete equações (A) e comparação entre $\mathrm{Kr}$ calculado com os valores das demais equações em função da cobertura do solo (B). 


\section{DEMANDA HÍDRICA DE FRUTEIRAS UTILIZANDO COEFICIENTE DE REDUÇÃO ADEQUADO AO PERÍMETRO IRRIGADO BAIXO ACARAÚ - CE}

$\mathrm{Na}$ Tabela 5 apresenta-se a evapotranspiração real (ETr) para as culturas estudadas durante o ano de 2010, utilizando o coeficiente de redução da evapotranspiração estimado pela equação encontrada. Pode-se observar que os maiores valores de ETr foram encontrados no mês de outubro. A graviola apresentou a maior demanda hídrica entre as culturas estudadas $\left(4,83 \mathrm{~mm} \mathrm{dia}^{-1}\right)$ enquanto a cultura da laranja apresentou os menores valores de $\operatorname{ETr}\left(1,99 \mathrm{~mm} \mathrm{dia}^{-1}\right)$. Ferreira et al. (2010) encontraram valores inferiores para a cultura da laranja $\left(1,05 \mathrm{~mm} \mathrm{dia}^{-1}\right) \mathrm{e}$ valores superiores para o pomar de manga $\left(3,08 \mathrm{~mm} \mathrm{dia}^{-1}\right)$ para o mês citado.

Tabela 5. Médias diárias da evapotranspiração de referência e evapotranspiração reduzida para diversas culturas no perímetro de irrigação do Baixo Acaraú - CE.

\begin{tabular}{|c|c|c|c|c|c|c|c|c|c|c|c|c|c|}
\hline \multicolumn{2}{|l|}{ Ano } & \multicolumn{12}{|c|}{2010} \\
\hline \multicolumn{2}{|c|}{ Meses } & jan & fev & $\operatorname{mar}$ & abr & mai & jun & jul & ago & set & out & nov & dez \\
\hline \multicolumn{2}{|c|}{$\begin{array}{c}\text { ETo } \\
\left(\mathrm{mm} \mathrm{dia}^{-1}\right)\end{array}$} & 3,76 & 4,22 & 3,75 & 3,56 & 3,84 & 4,16 & 4,94 & 5,70 & 6,14 & 6,30 & 6,10 & 5,62 \\
\hline Cultura & $\mathrm{Kr}$ & \multicolumn{12}{|c|}{$\mathrm{ETr}\left(\mathrm{mm} \mathrm{dia}{ }^{-1}\right)$} \\
\hline Coco & 0,48 & 1,71 & 1,92 & 1,70 & 1,62 & 1,76 & 1,92 & 2,27 & 2,61 & 2,82 & 2,90 & 2,81 & 2,59 \\
\hline Graviola & 0,90 & 2,85 & 3,20 & 2,83 & 2,70 & 2,93 & 3,19 & 3,79 & 4,35 & 4,70 & 4,83 & 4,68 & 4,32 \\
\hline Laranja & 0,39 & 1,17 & 1,32 & 1,16 & 1,11 & 1,21 & 1,31 & 1,56 & 1,79 & 1,93 & 1,99 & 1,92 & 1,78 \\
\hline Manga & 0,50 & 1,88 & 2,12 & 1,87 & 1,78 & 1,94 & 2,11 & 2,50 & 2,88 & 3,11 & 3,19 & 3,09 & 2,86 \\
\hline Sapoti & 0,63 & 1,64 & 1,84 & 1,63 & 1,55 & 1,69 & 1,84 & 2,18 & 2,51 & 2,71 & 2,78 & 2,70 & 2,49 \\
\hline
\end{tabular}

A Tabela 6 demonstra o volume de água (Va) a ser aplicado diariamente, para cada cultura em estudo. A partir dos resultados encontrados, constata-se que os maiores valores de volume de água a ser aplicado encontram-se nos meses do segundo semestre, em consequência da maior demanda evapotranspirativa das culturas. O mês de outubro apresenta a maior necessidade de água por parte das culturas estudadas, devendo-se dimensionar os sistemas de irrigação na região tendo como referência este mês, conforme afirmam Silva et al. (2010).

Frizzone \& Andrade Júnior (2005) afirmam que para a obtenção da produção máxima deve-se suprir a necessidade hídrica da cultura, mas ressaltam que os aspectos econômicos referentes à receita líquida devem ser estudados, pois os custos de operação e cultivo também devem ser levados em consideração no planejamento agrícola. Há uma necessidade de otimizar os sistemas de irrigação para se alcançar a máxima receita líquida, com estudos sobre o preço do produto e custos de energia elétrica como feito por Andrade Júnior et al. (2001), em estudos sobre estratégias ótimas de irrigação para a cultura da melancia. Frizzone (2007) afirma que a melhoria da eficiência do uso da irrigação não deve ser encarada apenas com $o$ manejo adequado da irrigação, mas com uma análise holística das fases pelas quais se passa todo o processo, desde a montagem do sistema de irrigação até a venda da produção agrícola. 


\section{DEMANDA HÍDRICA DE FRUTEIRAS UTILIZANDO COEFICIENTE DE REDUÇÃO ADEQUADO AO PERÍMETRO IRRIGADO BAIXO ACARAÚ - CE}

Tabela 6. Recomendação de irrigação para diversas culturas do perímetro irrigado do Baixo Acaraú CE.

\begin{tabular}{crrrrrrrrrrrr}
\hline & \multicolumn{10}{c}{2010} \\
\cline { 2 - 13 } Cultura & jan & fev & mar & abr & mai & jun & jul & ago & set & out & nov & dez \\
\cline { 2 - 13 } & \multicolumn{10}{c}{ Va $\left(\mathrm{L} \mathrm{dia}^{-1}\right)$} \\
\hline Coco & 32,8 & 36,8 & 32,5 & 31,0 & 33,7 & 36,7 & 43,5 & 50,0 & 54,1 & 55,6 & 53,8 & 49,7 \\
Graviola & 77,7 & 87,2 & 77,0 & 73,5 & 79,9 & 87,0 & 103,2 & 118,6 & 128,1 & 131,6 & 127,5 & 117,7 \\
Laranja & 10,9 & 12,3 & 10,9 & 10,4 & 11,3 & 12,3 & 14,5 & 16,7 & 18,1 & 18,6 & 18,0 & 16,6 \\
Manga & 36,8 & 41,4 & 36,5 & 34,9 & 37,9 & 41,3 & 49,0 & 56,2 & 60,8 & 62,4 & 60,5 & 55,8 \\
Sapoti & 20,3 & 22,8 & 20,2 & 19,2 & 20,9 & 22,8 & 27,0 & 31,0 & 33,5 & 34,5 & 33,4 & 30,8 \\
\hline
\end{tabular}

As culturas que apresentaram as maiores demandas hídricas anual foram respectivamente graviola, manga, coco, sapoti e laranja. Entretanto, deve-se resaltar que quando se comparam valores de volume a ser aplicado por planta a ordem muda em função do espaçamento da cultura. Os valores do consumo hídrico apresentado neste trabalho para as cultura da graviola e laranja estão acima dos valores apresentados por Ferreira et al. (2010), porém, dada as idades das plantas serem distintas em ambos os trabalhos, o consumo hídrico da planta provavelmente é diferenciado. Frazão \& Melo (2008) presumem que as anonáceas necessitam de 1000 a $1200 \mathrm{~mm} \mathrm{ano}^{-1}$, portanto, os valores apresentados no trabalho estão relativamente próximos dos recomendados.

Tabela 7. Demanda hídrica anual das diversas culturas no Perímetro irrigado do Baixo Acaraú - CE.

\begin{tabular}{|c|c|c|c|}
\hline \multirow[b]{2}{*}{ Cultura } & \multirow{2}{*}{$\begin{array}{c}\text { ETc } \\
\left(\mathrm{mm} \mathrm{ano}^{-1}\right)\end{array}$} & \multicolumn{2}{|c|}{$\mathrm{Va}$} \\
\hline & & $\left(\mathrm{L}\right.$ ano $\left.^{-1}\right)$ & $\left(\mathrm{m}^{3} \mathrm{ano}^{-1}\right)$ \\
\hline Coco & 810,2997 & $15.530,64$ & 15,53 \\
\hline Graviola & 1350,796 & $36.805,29$ & 36,80 \\
\hline Laranja & 555,0106 & $5.188,351$ & 5,19 \\
\hline Manga & 892,7429 & $17.458,92$ & 17,46 \\
\hline Sapoti & 778,1345 & $9.632,201$ & 9,63 \\
\hline
\end{tabular}

\section{CONCLUSÕES}

De acordo com o presente estudo, não existe diferença significativa entre as equações de $\mathrm{Kr}$ estudadas quando estas são aplicadas a culturas tropicais irrigadas por microaspersão. Assim sendo, a equação ajustada $\mathrm{Kr}=1,1226 . \mathrm{Cs}+0,0434$ mostrouse adequada para o manejo da irrigação na região do Baixo Acaraú. O período de maior demanda hídrica se encontra no segundo semestre do ano, devendo-se dimensionar os sistemas de irrigação com base neste período. 


\section{DEMANDA HÍDRICA DE FRUTEIRAS UTILIZANDO COEFICIENTE DE REDUÇÃ̃ ADEQUADO AO PERÍMETRO IRRIGADO BAIXO ACARAÚ - CE}

\section{REFERÊNCIAS BIBLIOGRÁFICAS}

ADECE. Agência de Desenvolvimento do Estado do Ceará. Governo do Estado do Ceará. Perímetros públicos irrigados do Ceará (2011). Disponível em: http://www.adece.ce.gov.br. Acesso em 15 de Dez de 2012.

AGRIANUAL. ANUÁRIO DA AGRICULTURA BRASILEIRA. São Paulo: FNP, 2007, 516p.

ANDRADE JÚNIOR, A. S.; FRIZZONE, J. A.; BASTOS, E. A.; CARDOSO, M. J.; RODRIGUES, B. H. N. Estratégias ótimas de irrigação para a cultura da melancia. Pesquisa Agropecuária Brasileira, v.36, n.2, p.301-305, 2001.

ALLEN, R. G.; PEREIRA, L. S. Estimating crop coefficients from fraction of ground cover and height. Irrigation Science, v.28, p. 17-34, 2009.

ALLEN, R.G.; PEREIRA, L.S.; RAES, D.; SMITH, M. Crop evapotranspiration Guidelines for computing crop water requirements. Roma: FAO, 1998. 300p. (FAO Irrigation and Drainage Paper, 56).

CARVALHO, M. A. R.; LEÃO, M. C. S.; CARVALHO, L. C. C.; SOUZA, F.; AGUIAR, J. V. Coeficientes de cobertura $(\mathrm{Kr})$ em Fruteiras Tropicais Adultas Microirrigadas. Revista Brasileira de Agricultura Irrigada, v.1, n.1, p.20-23, 2007.

DOGAN, E.; $\quad$ KIRNAK, H.; BEREKATOGLU, K.; BILGEL, L.; SUCURU, A. Water stress imposed on muskmelon (Cucumis Melo L.) with subsurface and surface drip irrigation systems under semi-arid climatic conditions. Irrigation Science, v.26, p.131-138, 2008.

DUTRA, I.; MEDEIROS, F. J.; PORTO FILHO, F. Q.; COSTA, M. C. Determinação do fator de cobertura do melão cultivado sob diferentes lâminas e salinidades da água de irrigação. Revista Brasileira de Engenharia Agrícola e Ambiental, v.4, n.2, p.146-151, 2000.

DOORENBOS, J.; PRUITT, J.O. Crop water requirement. Rome: FAO, 1977. 144p. FAO (Irrigation and Drainage Paper 24).

FERREIRA, T. T. S.; RODRIGUES, D. N. B.; GOMES FILHO, R. R. G. Demanda hídrica de fruteiras utilizando coeficiente de redução da evapotranspiração adequado a região do Baixo Jaguaribe no Ceará. Revista Brasileira de Agricultura Irrigada, v. 4, n.4, p.217-225, 2010.

FRANZÃO, A. A.; MELO, B. A cultura da acerola. Disponível em: $<$ http://www.fruticultura.iciag.ufu.br/acero la.htm> Acessado em: 5 abr. 2012

FRIZZONE, A. J. Planejamento da irrigação com uso de técnicas de otimização. Revista Brasileira de Agricultura Irrigada, v.1, n.1, p.24-49, 2007.

FRIZZONE, A. J.; ANDRADE JÚNIOR, A. S. Planejamento de irrigação: análise de decisão de investimento. Brasília: Embrapa, 2005, 656p.

FONTES, H. R.; FERREIRA, J. M. S.; SEIQUEIRA, L. A. Sistema de produção para a cultura do coqueiro. Brasília: Embrapa, 2002, 65p. (comunicado técnico).

GONDIM, R. S.; CASTRO, M. A. H.; EVANGELISTA, S. R. M.; TEIXEIRA, A. S.; FUCK JÚNIOR, S. C. F. Mudanças 


\section{DEMANDA HÍDRICA DE FRUTEIRAS UTILIZANDO COEFICIENTE DE REDUÇÃO ADEQUADO AO PERÍMETRO IRRIGADO BAIXO ACARAÚ - CE}

climáticas e impactos na necessidade hídrica das culturas perenes na Bacia do Jaguaribe, no Estado do Ceará. Pesquisa Agropecuária Brasileira, v.43, n.12, p.1657-1664, 2008.

GOMES, H.P. Engenharia de Irrigação: hidráulica dos sistemas pressurizados, aspersão e gotejamento. $2^{\mathrm{a}}$ edição. 390p. Universidade Federal da Paraíba, 1997.

HERA, M. L.; ROMERO, P.; PLAZA, E. G.; MARTINEZ, A. Is partial root-zone drying na effective irrigation technique to improve water use efficiency and fruit quality in field-grown wine grapes under semiarid conditions. Agricultural Water Management, v.87, p.261-274, 2007.

KLAR, A. E. Irrigação: frequência e quantidade de aplicação. São Paulo: Nobel, 1991. 156p.

KELLER, J.; KARMELI, D. Trickle Irrigation design Parameters. Transactions of the ASAE, St. Joseph, v. 17, n.4, p. 678-684, 1974.

MANTOVANI, E. C.; BERNARDO, S.; PALARETTI, L. F. Irrigação: princípios e métodos. $3^{\text {a }}$ Ed. Viçosa: Ed. UFV, 2009. $355 \mathrm{p}$.
PIZARRO CABELLO, F. P. Riegos

Localizados de Alta Frecuencia (RLAF)

- goteo - microaspersión - exudación. $3^{\text {a }}$

edición. Bilbao, Spain, 1996.

SILVA, A. N. P.; LIMA, F. J. L.; SILVA, A. O.; MOURA, G. B. A. Valores efetivos de precipitação pluvial para manejo da irrigação na cana-de-açucar em Goiana, Pernambuco. Revista Brasileira de Ciências Agrárias, v.5, n.4, p.585-591, 2010.

SILVA, J. A. A da. Características das anonáceas: Anonáceas, 2003. Disponível em:

http://www.todafruta.com.br/todafruta/mos tra_conteudo.asp?conteudo=2918. Acesso em: 03 Abr. 2012.

VIEIRA, S. Análise de variância: ANOVA. São Paulo: Atlas, 2006. 204p.

VERMEIREN, L.; JOBLING, G.A. Irrigação localizada. Tradução de H.R. Gheyi, F.A.V. Damasceno, L.G.A. Silva Jr.; J.F. de Medeiros, Campina Grande, UFPB, 1997. 184p. (Estudos FAO: Irrigação e Drenagem, 36). 